Laboratory Investigations

\title{
Ultrasound-guided dissection and ligation of the internal inguinal ring for hernia repair in pediatrics: an experimental animal study ${ }^{\text {th }}$
}

\author{
Pedro Reino-Pires a,b,c,* José Miguel Pêgo a,b ${ }^{\text {a, }}$, Alice Miranda ${ }^{\text {a,b }}$, Catarina Barroso ${ }^{\text {a,b,d }}$, \\ Margarida España ${ }^{c}$, Jorge Correia-Pinto ${ }^{\text {a,b,d }}$ \\ a Life and Health Sciences Research Institute (ICVS), School of Medicine, University of Minho, 4710-057, Braga, Portugal \\ b ICVS/3B's - PT Government Associate Laboratory, 4710-057, Braga/Guimarães, Portugal \\ c Hospital de Dona Estefânia Hospital de Dona Estefânia, Serviço de Cirurgia Pediátrica, Rua Jacinta Marto, 1169-045 Lisbon, Portugal \\ d Department of Paediatric Surgery, Hospital de Braga, 4710-243, Braga, Portugal
}

\section{A R T I C L E I N F O}

\section{Article history:}

Received 26 October 2016

Received in revised form 1 February 2017

Accepted 21 March 2017

\section{Key words:}

Minimally invasive

Surgery

Laparoscopy

Rabbits

Ultrasound

\begin{abstract}
A B S T R A C T
Purpose: We aimed to test the feasibility and reliability of ultrasound-guided percutaneous internal inguinal ring suture in rabbits, as a model for inguinal hernia repair in pediatric population.

Methods: Twenty-eight rabbits were divided in 2 groups: group I (female morphology) - persistence of the peritoneal-vaginal duct with gonads placed in intraperitoneal position; group II (male morphology) - persistence of the peritoneal-vaginal duct with gonads kept intact inside the duct. Under exclusive ultrasound-guided image we tried to perform a complete pre-peritoneal ligation of the peritoneal-vaginal duct at the level of the internal inguinal ring using a 20G peripheral IV catheter and 2-0 non-absorbable suture. Afterwards, an exploratory laparoscopy was performed to evaluate the ligation.

Results: Ultrasound allowed characterization of inguinal-crural structures. Group I - complete and reliable suture $66.7 \%$, incomplete but reliable suture $16.7 \%$, inappropriate ligation $16.7 \%$; group II - complete but unreliable suture $76.9 \%$, incomplete and unreliable suture $11.5 \%$, inappropriate suture $11.5 \%$. No acute complications were logged. Conclusions: Percutaneous dissection and ligation of internal inguinal ring through exclusive ultrasound guidance was feasible and likely reliable, namely for female inguinal hernia repair.
\end{abstract}

(c) 2017 Elsevier Inc. All rights reserved.
Since the dawn of laparoscopy, minimally invasive surgery has been spreading worldwide. As the techniques evolve, new strategies have been applied, as the use of natural orifices, robotically assisted procedures and percutaneous laparoscopic assisted procedures, in a quest to be even less invasive. While the open techniques for indirect inguinal hernia correction, a common procedure in pediatric surgery, are well established and have had their own evolution, laparoscopic correction has emerged in this global context [1,2], peaking nowadays for some into a scar less percutaneous procedure [3].

Simultaneously, the ultrasound technology has evolved greatly, with the technology becoming more available and being currently used in areas other than radiology and diagnostics. The ultrasound-guided loco-regional anesthesia is a well-established example of the ultrasound utility in a surgical setting [4,5]. Likewise, ultrasound imaging is for some the diagnostic exam for a groin hernia $[6,7]$ as well as a resource that can be accurately used by a surgeon as a diagnostic tool $[8,9]$.

\footnotetext{
it Conflict of interests: none.

* Corresponding author at: Serviço de Cirurgia Pediátrica, Hospital de Dona Estefânia, Rua Jacinta Marto, 1169-045 Lisbon, Portugal. Tel.: + 351213126898. E-mail addresses: pedro.pires@chlc.min-saude.pt, pedro.reino.pires@gmail.com (P. Reino-Pires).
}

Aiming for an even less invasive procedure, based in the laparoscopic assisted procedure experience, we postulate that an ultrasoundguided internal ring suture (UGIRS) could be a reproducible, feasible, reliable and safe procedure for indirect inguinal hernia in pediatric female patients, avoiding the inherent risks of an abdominal cavity entry and pneumoperitoneum. Therefore we conducted this study in an animal model aiming to prove this concept. To accomplish this, we performed an UGIRS in a living rabbit model using a 20G peripheral IV catheter and a $2 / 0$ monofilament non-absorbable suture, dividing the study into two groups, being the first a simulation of the human female indirect inguinal hernia and the second a simulation of the male's morphology. To our knowledge, this is the first study that evaluates the role of ultrasound imaging in percutaneous repair of inguinal defects.

\section{Materials and methods}

The New Zealand male rabbit was the selected animal model since it has a patent processus vaginalis [10] where an inguinal ring (IR) suture can be performed [11]. Also, this is a low cost animal model, with a comparable size to the newborn, making it a popular model for surgical training. Moreover its anatomy is well known, particularly the main landmarks of inguinal ultrasound evaluation $[12,13]$. The relation between these landmarks are different than in the human, nevertheless 


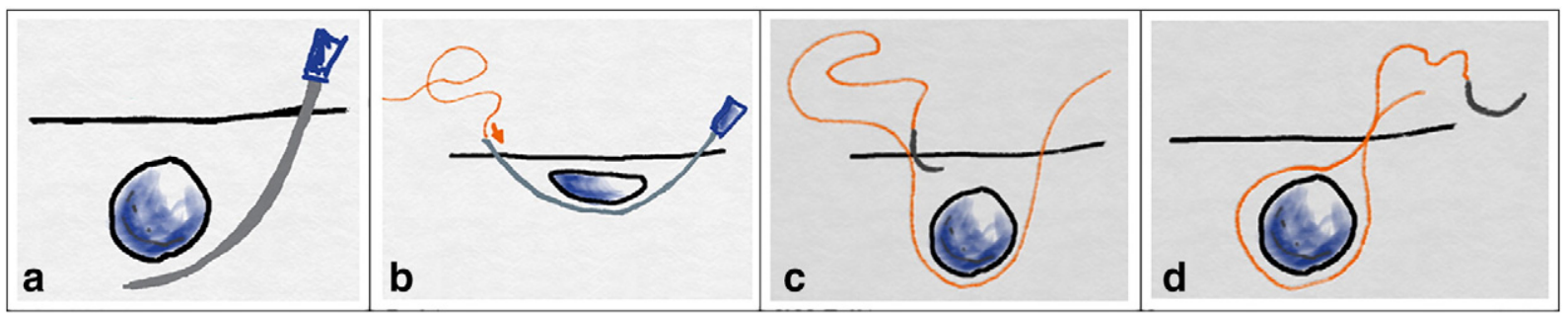

Fig. 1. Schematics of procedure's main steps.
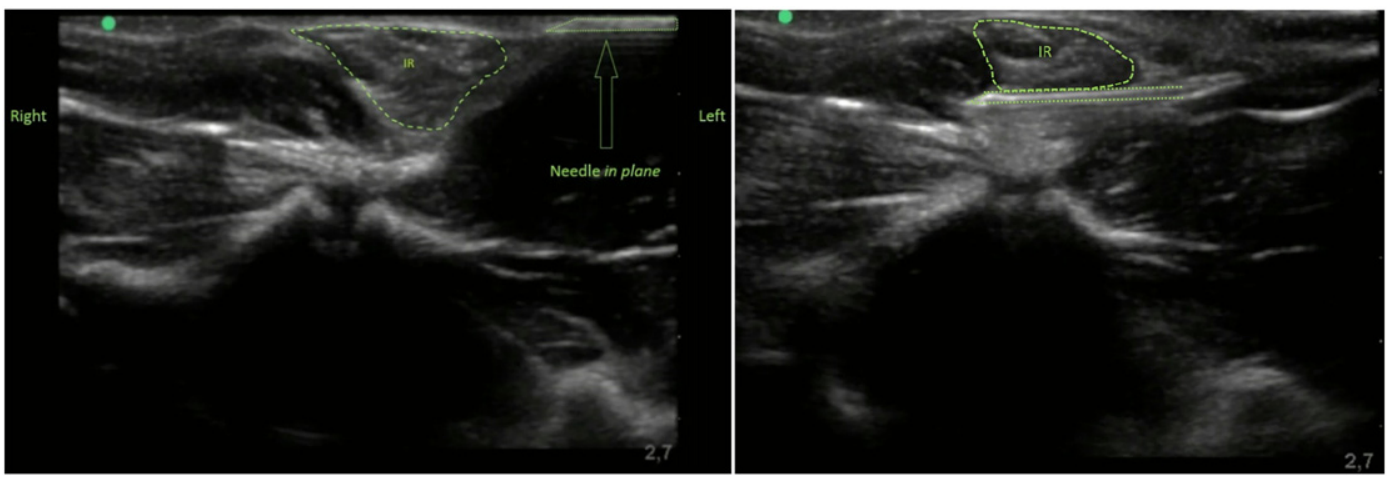

Fig. 2. Ultrasound stills during in plane needling technique of UGIRS.

our study aims to evaluate an exclusively ultrasound-guided percutaneous suture of the internal inguinal ring, placed in an extraperitoneal position; therefore we considered this model suited for our purpose.

Under general anesthesia (ketamine $25 \mathrm{mg} / \mathrm{kg}+$ medetomidine $0,3 \mathrm{mg} / \mathrm{kg}$ subcutaneous), the rabbit is submitted to trichotomy and placed in Trendelenburg position. In the first group of the study, testes are placed in an intraperitoneal position through a laparoscopic approach using a 5$\mathrm{mm} 30^{\circ}$ laparoscope medially and 3-mm scissor and grasper laterally on each side, leaving the patent processus vaginalis void of content and simulating the female Nuck's duct. Pneumoperitoneum is evacuated. In the second group no further manipulation was done prior to the UGIRS. Using a 13-MHz linear ultrasound probe, anatomical landmarks are identified (inferior epigastric artery emergence, ileofemoral vessels, inguinal ligament, patent processus vaginalis and IR) and the duration of this step recorded.

With a previously bended $20 \mathrm{G}$ peripheral IV catheter, the rim of the IR is punctured laterally and the needle oriented under the IR from side to side, circumscribing the IR (Fig. 1a). The puncture is done under exclusive ultrasound visualization using an in plane needling technique, with the probe fixed over the IR in a transverse plane (Fig. 2). The end of a monofilament non-absorbable $2 / 0$ suture is inserted through the catheter's lumen (Fig. 1b) and the latter removed. The suture's needle is then oriented subcutaneously back from the skin's second puncture site to the first (Fig. 1c and d) and secured with a mosquito forceps.

The time of the procedure is logged. A second landmark identification and UGIRS are performed in the contralateral inguinal region, in the same fashion. A 5-mm $30^{\circ}$ laparoscope is then placed through a medial port and a 3-mm grasper inserted through a stab-wound. At this stage, for each side, the IR suture success is verified and catalogued within the previously established categories: complete and reliable ligation (Fig. 3), incomplete but reliable ligation (Fig. 4), complete but unreliable ligation (Fig. 5), incomplete and unreliable ligation (Fig. 6), inappropriate ligation - the latter regarding distal ligations without closing the IR or with a major gap. To better appreciate the ligation result, the knot was loosened and tightened during exploratory laparoscopy, in a dynamic fashion. (See Figs. 7 and 8.)

Complications and organ damage were also searched. Note that the procedure results are verified in a dynamic fashion, with the knot tied and loosened. After the completion of both the procedures and
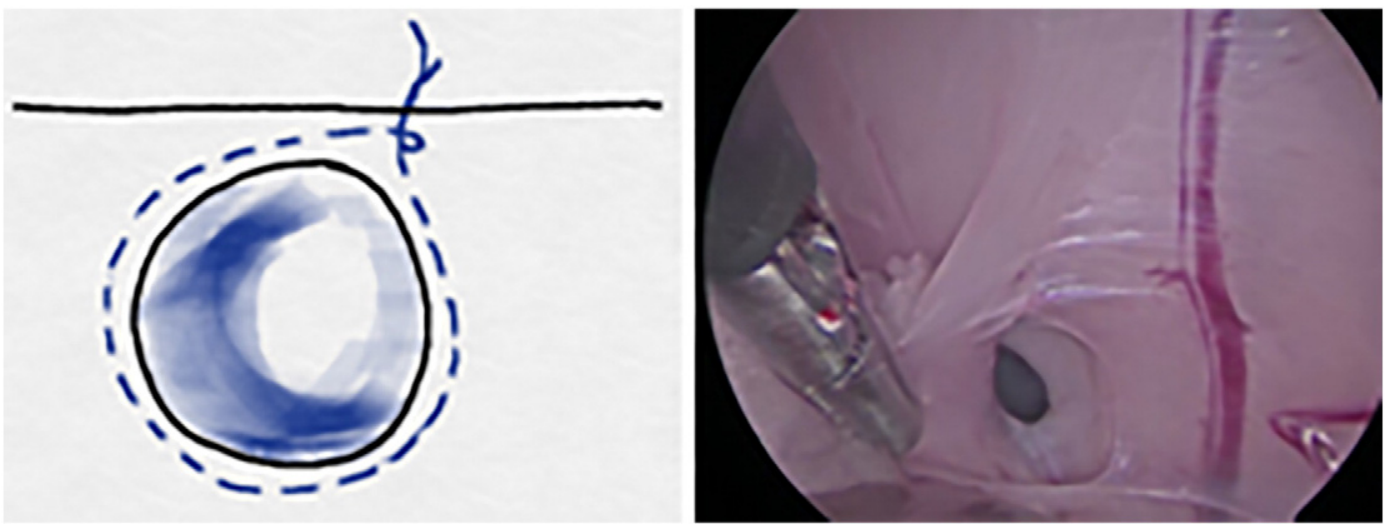

Fig. 3. Complete and reliable ligation schematics and laparoscopy photo. No gap and without spermatic cord entrapment. 

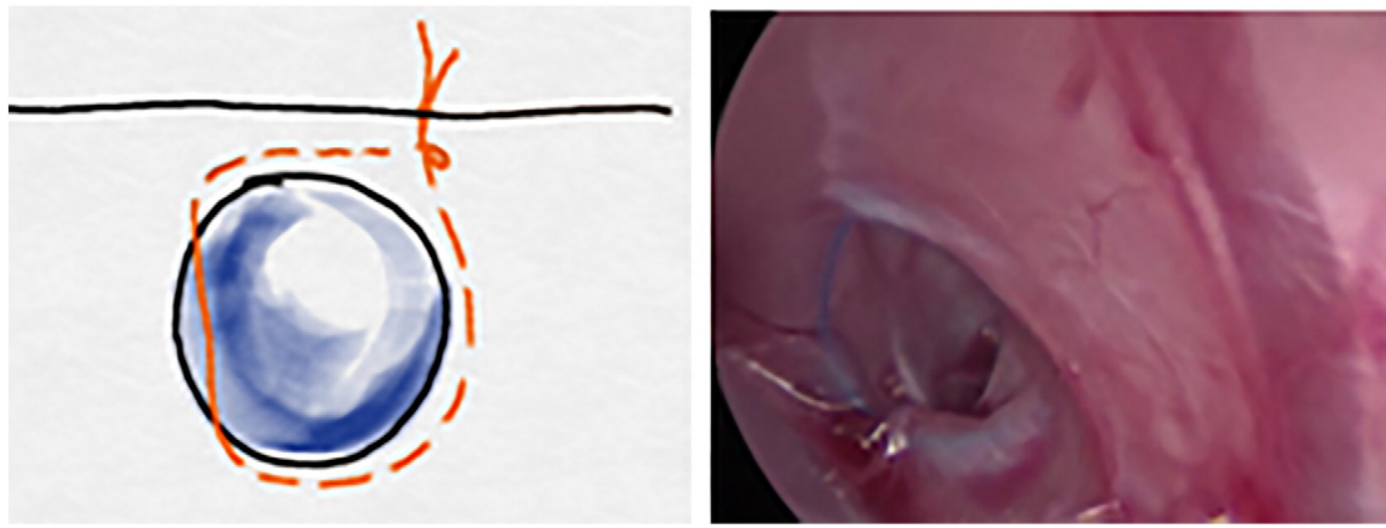

Fig. 4. Incomplete but reliable ligation schematics and laparoscopy photo. With minor gap and without spermatic cord entrapped.
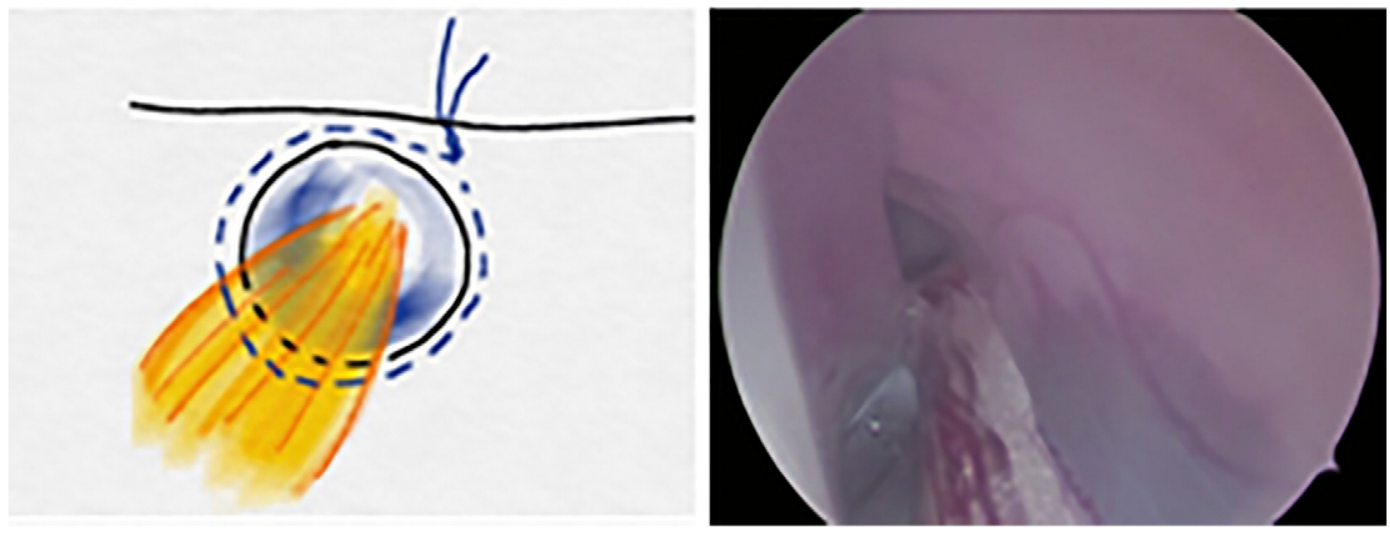

Fig. 5. Complete but unreliable ligation schematics and laparoscopy photo. No gap but with spermatic cord entrapped.
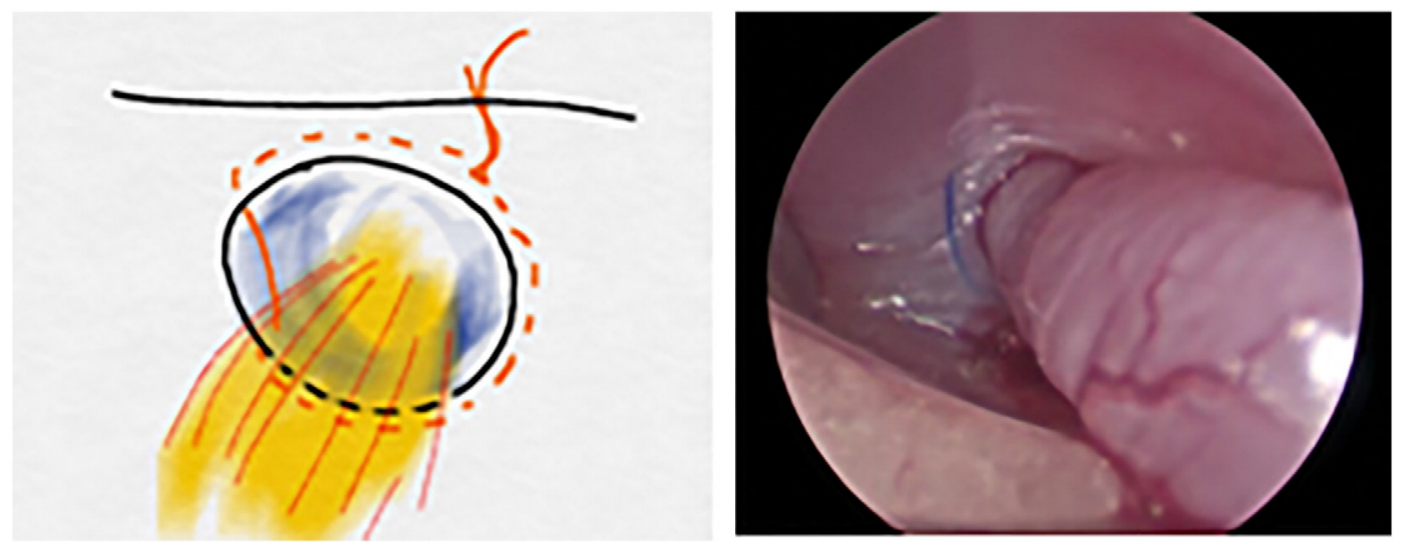

Fig. 6. Incomplete and unreliable ligation schematics and laparoscopy photo. With minor gap and spermatic cord entrapped.

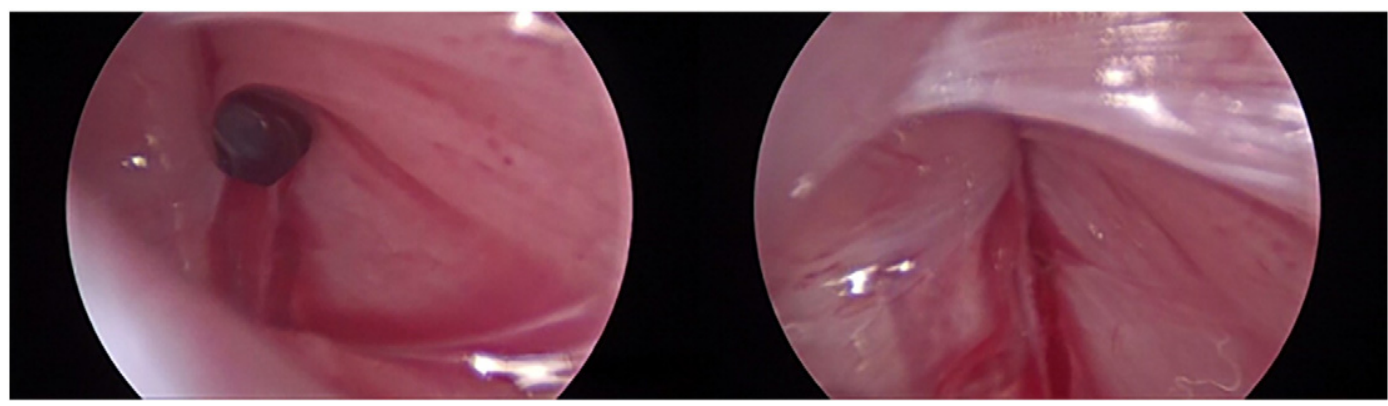

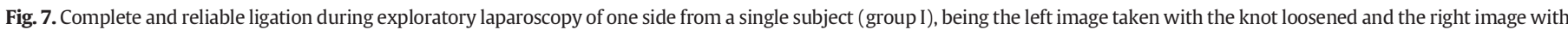
the knot tied. 


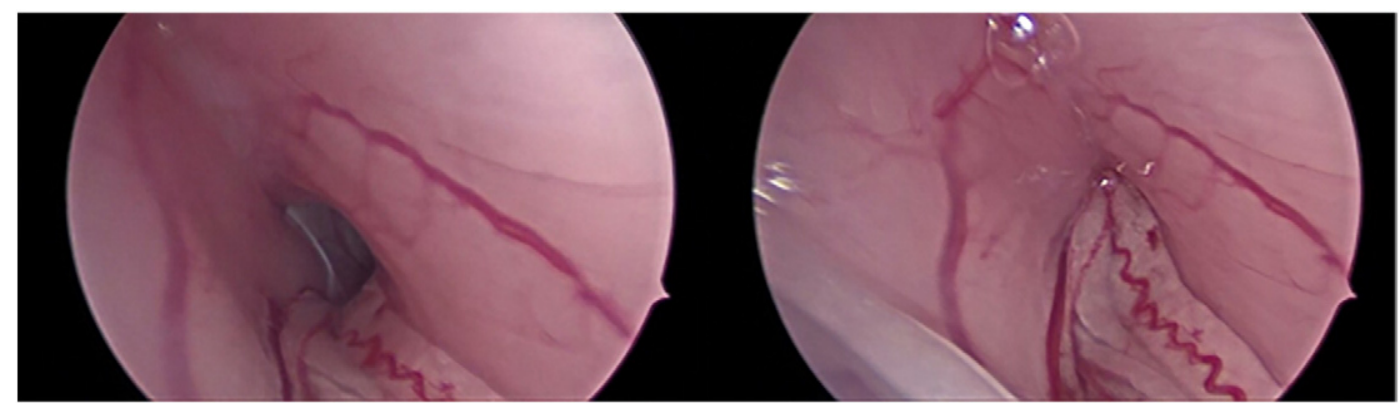

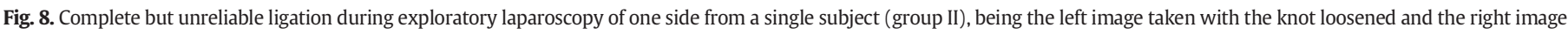
with the knot tied.

exploratory laparoscopy, the rabbits were euthanized (penthobarbital $150 \mathrm{mg} / \mathrm{kg}$ intravenous).

\section{Results}

Twenty-eight rabbits were included in the study with an average weight of $2.3 \mathrm{~kg}$, corresponding to a total of 30 UGIRS performed in the first group and 26 UGIRS in the second. Results are summarized in Table 1.

In group I, the landmark identification was successful in all 30 procedures, with a mean time of $2.6 \mathrm{~min}$ ( $\min .1$, max. 5). The mean time of the procedure itself was $4.6 \min$ ( $\min .2$, max. 7). Laparoscopy confirmed a complete ligation in 20 cases (66.7\%), as seen in Fig. 1, and an incomplete but reliable ligation in 5 cases (16.7\%). Inappropriate ligation was verified in 5 cases (16.7\%). No complications were recorded.

In group II, the landmark identification was successful in all 26 procedures, with a mean time of $6.2 \mathrm{~min}$ (min. 2, max. 10). The mean time of the procedure itself was $5.2 \mathrm{~min}$ ( $\min .1$, max. 18). Laparoscopy confirmed complete but unreliable ligation in 20 cases (76.9\%), as shown in Fig. 2, incomplete and unreliable ligation in 3 cases (11.5\%), and inappropriate ligation in 3 (11.5\%). There were logged 2 cases of spermatic cord transection, regarded as incomplete ligations. No other complications occurred.

\section{Discussion}

The indirect inguinal hernia treatment evolved from open approaches to a percutaneous laparoscopic procedure. The gold standard treatment is yet to be established but we believe it to be associated with a laparoscopic approach since it provides the opportunity to screen for a metachronous inguinal hernia as well as it can be performed as an outpatient procedure in a scarless fashion. However, we also believe that the evolution has not ceased, as there is always place to perfect a known technique. Likewise, ultrasonography has evolved greatly, as mentioned above. It is in our belief that an ultrasonography evolution - better probes, better ultrasound software, ultrasound dedicated instruments and selective ultrasound contrasts - may allow us to perform further more complex ultrasound-guided procedures, hypothesizing its use in dissection, ligation, cut and cauterization, potentially climaxing in even less-invasive procedures.

This study aimed to verify the reliability and security of a reproducible ultrasound-guided inguinal hernia repair, designed with the female's pediatric population in mind. Following that train of thought, and to better simulate the Nuck's duct, the rabbit's testis had to be placed in an intraabdominal position prior to the UGIRS execution. Hypothesizing that this abdominal cavity violation and abdominal content manipulation could avoid UGIRS complications, we also performed the procedure without previous manipulation and therefore simulating a male morphology. However, it is important to mention that the spermatic cord of the rabbit passes almost freely through the duct's lumen, so the cord's entrapment was expected in the second group's ligations. Because of this detail, the cord's entrapment was not interpreted as a complication but rather as an unreliable ligation. Considering this relevant aspect and that the sought result was an extraperitoneal ligation of the inguinal canal in relation with the IR, complete or with minor gap, one can assume that UGIRS was successful in $83.3 \%(n=25)$ of the cases in the first group and in $88.5 \%(n=23)$ in the second.

The major difficulties of this model were related to the small model's size, namely the identification of the inferior epigastric vessels and of the exact puncture site in the study's first group. The latter also was more difficult because of the anatomical relations between our ultrasound landmarks and sometimes because of conflict of space of the animal's lower limb with the probe. Anyway, we have shown that a complete ligation is possible with UGIRS, therefore theoretically this technique competes with other known minimally invasive procedures such as the percutaneous approaches. Like those, a metachronous hernia diagnosis and herniated content reduction confirmation is possible with an ultrasound scan, since this technique is suited for the purpose and might be performed by a surgeon as previously mentioned. The scan could be done before anesthesia, when a Valssalva maneuver could help the hernia identification, and in the moment of the procedure. Therefore, UGIRS comes with some major advantages in comparison. With this method there is no need to a peritoneal cavity entry, since the ligation is performed in an extraperitoneal plane and a laparoscopy is not required. Also, the suture is executed in a plane over the inguinal ligament, protecting the ileal vessels and so lowering the inherent haemorrhagic and vascular lesion risks. Consequently, considering a careful IR identification, a safe in plane needling technique and a suture containing the small peritoneum fold that exists under the round ligament, we believe that this procedure might be safely performed by surgeons in human females, with a combination of local anesthesia and sedation. In order to progress to clinical practice, we believe that some technological development is needed. Similarly to the needles

Table 1

Summary of results.

\begin{tabular}{|c|c|c|}
\hline & Group I (female morphology) & Group II (male morphology) \\
\hline Complete and reliable ligation, $\mathrm{n}(\%)$ (no gaps and without spermatic cord entrapment) & $20(66.7 \%)$ & 0 \\
\hline Incomplete but reliable ligation, $\mathrm{n}(\%)$ (with minor gap and without spermatic cord entrapped) & $5(16.7 \%)$ & 0 \\
\hline Complete but unreliable ligation, $\mathrm{n}(\%)$ (no gaps but with spermatic cord entrapped) & - & $20(76.9 \%)$ \\
\hline Incomplete and unreliable ligation, $\mathrm{n}(\%)$ (with minor gaps and spermatic cord entrapped) & - & $3(11.5 \%)$ \\
\hline Inappropriate ligation, $\mathrm{n}(\%)$ (distal ligation or with major gap) & $5(16.7 \%)$ & $3(11.5 \%)$ \\
\hline
\end{tabular}

Note that since there is no spermatic cord passing through the inguinal canal in the first group, there were only considered the three possible categories. 
used in loco-regional anesthesia, a 20G needle with proper echogenic properties and toughness prone for bending would be important. Also, an echogenic suture would help the US placement and control of the ligation. Considering an eventual technology evolution, this procedure could be even performed in human males. In this hypothetic setting, the US with Doppler imaging would allow to evaluate the testis vascularization after the procedure, lowering the risk of ischemia. Unfortunately, to our knowledge, there is no animal model of the indirect inguinal hernia in males that would allow us to test this possibility.

This study tested and demonstrated the potential of ultrasonography in surgical procedures, creating a new perspective of the concept of minimally invasive surgery that, in this particular case and even though further studies are warranted, can be almost immediately ranslated to the indirect inguinal hernia repair in the pediatric female population.

\section{Acknowledgements}

This work was supported by the Fundação para a Ciência e Tecnologia (FCT), co-funded by Programa Operacional Regional do Norte (ON.2-O Novo 267 Norte); from the Quadro de Referência Estratégico Nacional (QREN) through the Fundo Europeu de Desenvolvimento Regional (FEDER) and from the Projeto Estratégico - LA 26 - 2013-2014 (PEst-C/ SAU/LA0026/2013).

Alice Miranda has an individual FCT fellowship (SFRH/BD/52059/2012).

Pedro Reino-Pires would like to thank the support of Dr. João Pascoal, Director of the Pediatric Surgery Department of Hospital de Dona Estefânia.

\section{Appendix A. Supplementary data}

Supplementary data to this article can be found online at http://dx. doi.org/10.1016/j.jpedsurg.2017.03.057.

\section{References}

[1] Montupet P, Esposito C, Roblot-Maigret B. Laparoscopic treatment of congenital inguinal hernia in children. J Pediatr Surg 1999;34(3):420-3.

[2] Bertozzi M, Marchesini L, Tesoro S, et al. Laparoscopic herniorrhaphy in children. Pediatr Med Chir 2015;28:37(2).

[3] Patkowski D, Czernik J, Chrzan R, et al. Percutaneous internal ring suturing: a simple minimally invasive technique for inguinal hernia repair in children. J Laparoendosc Adv Surg Tech A 2006;16(5):513-7.

[4] Mariano ER, Marshall Z], Urman RD, et al. Ultrasound and its evolution in perioperative regional anesthesia and analgesia. Best Pract Res Clin Anaesthesiol 2014;28(1):29-39.

[5] Muhly WT, Orebaugh SL. Ultrasound evaluation of the anatomy of the vessels in relation to the femoral nerve at the femoral crease. Surg Radiol Anat 2011;33:491-4.

[6] Lee RKL, Griffith JF, Ng AWH. High accuracy of ultrasound in diagnosing the presence and type of groin hernia. J Clin Ultrasound 2015;43:538-47.

[7] Robinson A, Light D, Nice C. Meta-analysis of sonography in the diagnosis of inguinal hernias. J Ultrasound Med 2013;32:339-46.

[8] Burford JM, Dassinger MS, Smith SD. Surgeon-performed ultrasound as a diagnostic tool in appendicitis. J Pediatr Surg 2011;46(6):1115-20.

[9] Rozycki GS. Surgeon-performed ultrasound: its use in clinical practice. Ann Surg 1998;228(1):16-28.

[10] Lossi L, D'Angelo L, De Girolamo P, et al. Anatomical features for an adequate choice of experimental animal model in biomedicine: II. Small laboratory rodents, rabbit, and pig. Ann Anat 2015;204:11-28.

[11] Kelly KB, Krpata DM, Blatnik JA, et al. Suture choice matters in rabbit model of laparoscopic, preperitoneal, inguinal hernia repair. J Laparoendosc Adv Surg Tech A 2014;24(6):428-31.

[12] Pérez W, Möller R, Martin E. Peritoneal folds of the rabbit (Oryctolagus cuniculus). Anat Histol Embryol 2005;34:167-70.

[13] Bavaresco AZ, Culau POV, Campos R. Parietal collateral and terminal groups of the abdominal aorta in New Zealand rabbits (Oryctolagsus cuniculus). Acta Sci Vet 2012;40(4):1069. 